\title{
Semiconductor nanowire solar cells: synthetic advances and tunable properties
}

\section{Citation}

Kempa, Thomas J., and Charles M. Lieber. 2014. "Semiconductor Nanowire Solar Cells: Synthetic Advances and Tunable Properties." Pure and Applied Chemistry 86 (1) (January 22). doi:10.1515/pac-2014-5010.

\section{Published Version}

10.1515/pac-2014-5010

\section{Permanent link}

http://nrs.harvard.edu/urn-3:HUL.InstRepos:33464194

\section{Terms of Use}

This article was downloaded from Harvard University's DASH repository, and is made available under the terms and conditions applicable to Other Posted Material, as set forth at http:// nrs.harvard.edu/urn-3:HUL.InstRepos:dash.current.terms-of-use\#LAA

\section{Share Your Story}

The Harvard community has made this article openly available.

Please share how this access benefits you. Submit a story.

Accessibility 


\title{
Conference paper
}

Thomas J. Kempa* and Charles M. Lieber

\section{Semiconductor nanowire solar cells: synthetic advances and tunable properties ${ }^{1}$}

\begin{abstract}
The solar power received by Earth far exceeds global power demands. Despite this, infrastructure shortages and high capital costs prevent the wide-scale adoption of photovoltaics to displace conventional energy technologies relying on carbon-based fuels. In response, new concepts and materials have been explored to develop next-generation solar cells capable of operating more efficiently and cheaply. Over the past decade, single semiconductor nanowire (NW) and NW array devices have emerged as promising platforms with which to examine new concepts. Small distances in NWs allow for efficient charge separation while tunable photonic modes permit light absorption properties distinct from bulk materials. Furthermore, the synthesis and fabrication of NW devices presents new opportunities such as with incorporation of complex heterostructures or use of cheaper substrates. Here, we present a critical discussion of the benefits and remaining challenges related to utilization of NWs for solar energy conversion and emphasize the synthetic advances leading towards significant improvement in the electrical and optical performance of NW devices. We conclude by articulating the unique capabilities of solar cells assembled from multiple, distinct NWs.
\end{abstract}

Keywords: nanowires; photonics; solar energy; synthesis.

${ }^{1}$ A collection of invited, peer-reviewed articles by the winners of the 2013 IUPAC Prize for Young Chemists.

*Corresponding author: Thomas J. Kempa, Department of Chemistry and Chemical Biology, Harvard University, Cambridge, MA 02138, USA, e-mail: tkempa@fas.harvard.edu

Charles M. Lieber: Department of Chemistry and Chemical Biology, Harvard University, Cambridge, MA 02138, USA

\section{Introduction}

Semiconducting nanowires (NWs) are a remarkably versatile class of nanomaterial (Fig. 1) that have enabled a diverse range of devices for nanoelectronics, biosensing, photonics, and energy science [1-14]. This review discusses recent synthetic advances that have expanded the functionality and complexity of NW building blocks for a new generation of photovoltaic devices. We focus on the synthetically tunable electrical and optical properties of these structures and their promise as a platform for efficient solar cells.

\section{Overview of nanowire synthesis}

Semiconductor NWs are typically synthesized using metal nanoclusters as catalysts during a vapourliquid-solid (VLS) growth process [15]. In this process (Fig. 2), a vapor-phase semiconductor source dissolves in the liquid metal/semiconductor droplet maintained at a temperature above the metal/semiconductor eutectic point. Continued supply of the semiconductor gaseous reactant supersaturates the droplet, whereupon the chemical potential between the gas-liquid and liquid-solid interfaces acts as a driving force for nucleation of the solid semiconductor. Ultimately, the liquid-solid interface acts as a sink leading to continued semiconductor incorporation into the lattice and growth of the NW with the droplet carried at the tip of the incipient NW. 


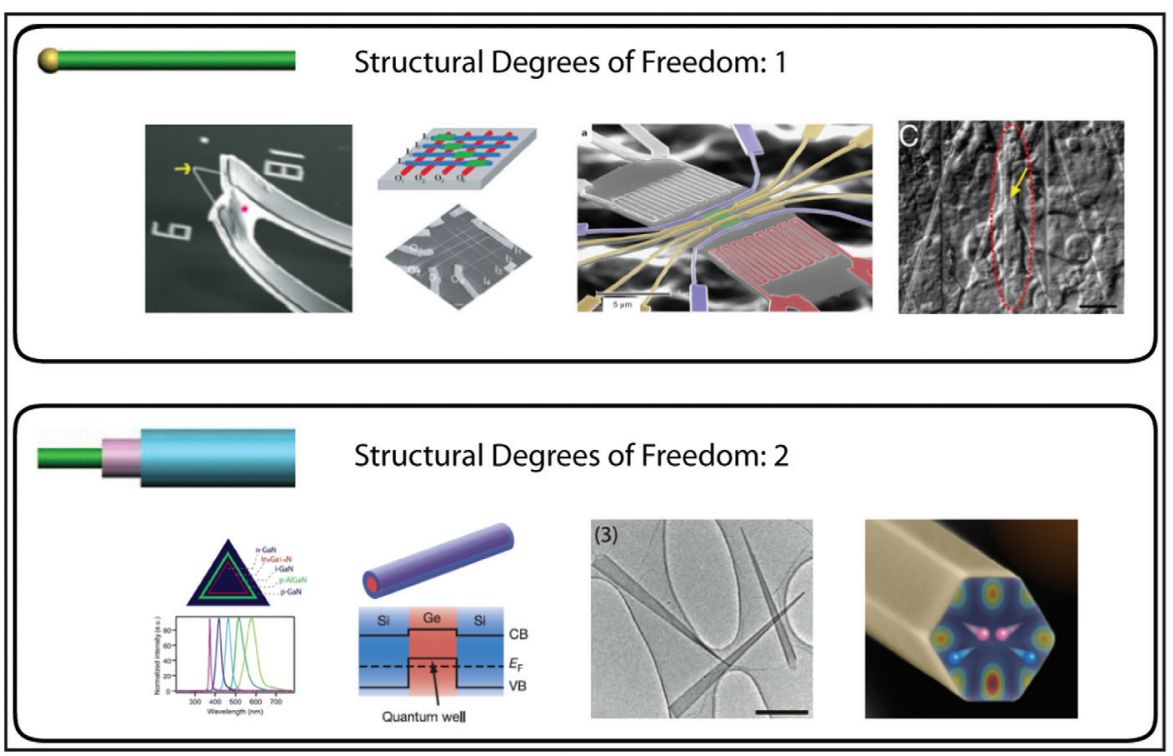

Fig. 1 Semiconductor NWs and their applications. Nanoscale solar cells, photonic elements, and biosensors have been demonstrated.

Gaseous semiconductor reactants are typically generated through thermal or plasma-enhanced decomposition of precursors such as in a chemical vapor deposition (CVD) or plasma-enhanced chemical vapor deposition (PECVD) process. Alternate methods involve momentum or energy transfer such as in pulsed laser ablation [17] or molecular beam epitaxy (MBE) [18] from solid targets. Despite a range of alternatives, CVD has emerged as the most popular technique. In the case of Si nanowire (SiNW) growth, silane $\left(\mathrm{SiH}_{4}\right)$ and $\mathrm{Au}$ nanoparticles are normally used as the precursor and catalyst, respectively. In addition to group IV materials, compound III-V and II-VI NWs have been produced with the VLS method, employing as a guide to choice of synthetic parameters (e.g., partial pressure, temperature) the pseudobinary phase diagrams for the catalyst and compound semiconductor of interest. For compound semiconductor growth, metal-organic chemical vapor deposition (MOCVD) [20] or pulsed laser ablation [21] have typically been used to deliver gaseous precursors.

Despite a simple reactor set-up (described previously in [16]) and relatively narrow range of synthetic parameters (e.g., temperature, reactant partial pressure, diluent or carrier gas, and metal catalyst), CVD still enables growth of a broad range of NW materials. It is possible to grow axial heterostructures (Fig. 3) in which sections of different materials or like materials with different doping are grown along the wire axis [22, 23], and, more recently, kinked axial structures have been demonstrated [24]. Radial heterostructures (Fig. 3) in

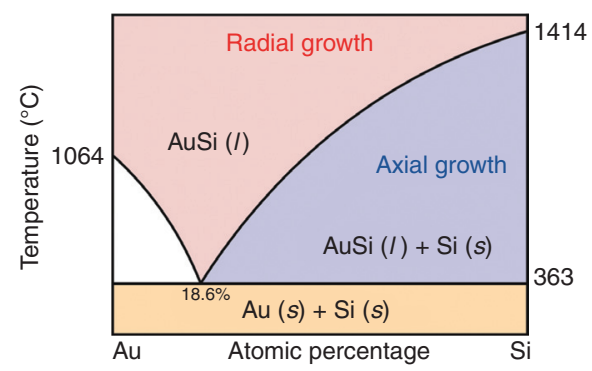

Fig. 2 Au-Si phase diagram. The Au-Si binary phase diagram describes the temperature and Si concentration (partial pressure) required to maintain a Au-Si eutectic that is crucial for sustaining VLS-growth of axial NWs. Deposition of Si from the vapor phase predominates at higher temperatures and lower Si partial pressures. Reprinted with permission from ref. [16]. Copyright (c) 2009, B. P. Timko. 


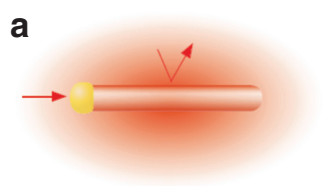

C

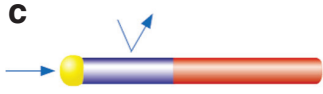

e

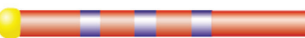

b

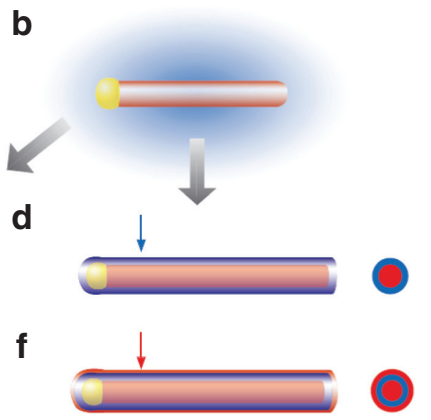

Fig. 3 NW heterostructure synthesis. (a) Reactant incorporation at catalyst (growth end) leads to one-dimensional axial growth; (b) change of reactant leads to axial or radial NW heterostructure growth; (c) axial growth: if reactant is preferentially incorporated at the catalyst; (d) radial growth: if reactant is uniformly incorporated on the wire surface; (e) modulation of reactants can produce axial superlattices; (f) modulation of reactants produces multishell structures. Reprinted with permission from ref. [19]. Copyright (c) 2004, Royal Society of Chemistry.

which the VLS grown core is subsequently sheathed by a single or multiple shells of semiconductor material [12-14, 25, 26] have been demonstrated.

\section{Nanowires in photovoltaics}

Next-generation solar cells, which are also referred to as third-generation solar cells, aim to achieve $>20 \%$ solar-to-electric power conversion efficiencies at a specific cost of $<$ USD0.50 per peak Watt ${ }^{1}$ [27]. Over the past decade an intensive research effort was launched to study how NW materials could contribute to achieving this goal. Several hypotheses were motivating research in this direction. The first of these was that low-temperature, high-purity, and high-yield synthesis of nanomaterials could reduce costs compared to conventional top-down fabrication, which typically entails high thermal budgets and complex processes to make the high-purity materials necessary for solar cell applications [27-29]. The second hypothesis is that increased optical absorption in nanomaterials, due to unique properties of light interaction with subwavelength objects and periodic structures, could increase efficiencies beyond those achieved on thick semiconductor films [30]. In addition, previous research [31,32] has shown that integration of heterogeneous materials (e.g., semiconductors with $>4 \%$ lattice mismatch) is more readily possible at the nanoscale and could thus enable further efficiency enhancements through improved absorption. The third hypothesis is that nanomaterials could be used to explore new material combinations or to exploit physical effects such as hot carriers, multiple excitons, or intermediate bands to achieve efficiencies beyond the Shockley-Queisser efficiency [33-37].

Many groups have investigated semiconductor NWs and how they could enhance photovoltaic or photoelectrochemical power conversion efficiencies. Using hydrothermally grown $\mathrm{ZnO}$ NWs decorated with a Ru-based dye and immersed in electrolyte (Fig. 4a), a NW dye-sensitized photoelectrochemical solar cell showed improved current density compared to $\mathrm{ZnO}$ nanoparticle-based dye-cells for the same dye loading level [38]. The authors argued that the $\mathrm{ZnO} \mathrm{NW}$ photoanode increases both the productive absorption and efficiency of carrier extraction compared to $\mathrm{ZnO}$ nanoparticles due to improved charge percolation pathways. Work from the Atwater and Lewis groups has led research investigating the performance of periodic arrays of Si microwires (Fig. 4b) for photovoltaic and solar-to-fuel conversion systems [39-41]. Garnett

1 This quantity reflects the cost of generated energy per peak Watt $\left(\mathrm{W}_{\mathrm{p}}\right)$ for purposes of comparing against other energy generation platforms. Care should be taken to consider capital and installation costs, balance of system costs, fuel costs (where applicable), and maintenance over the life of the generator. A peak Watt is defined as the peak power under ideal illumination conditions. 
a

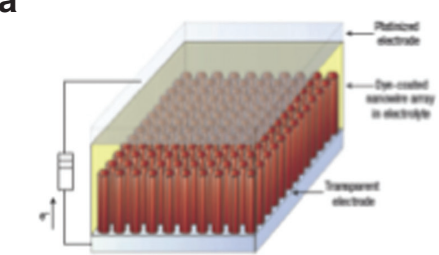

C

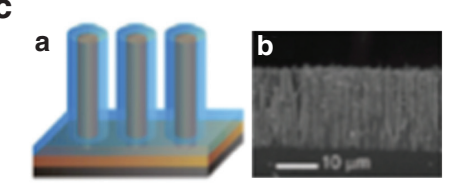

d

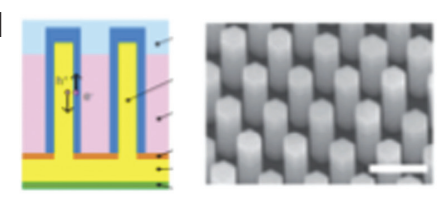

e

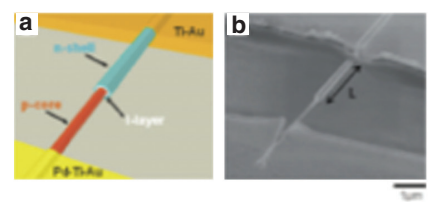

b

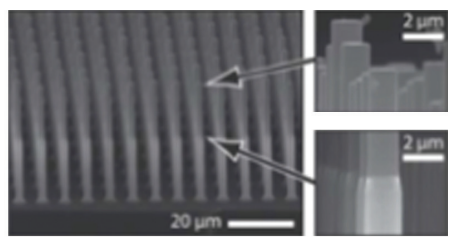

f

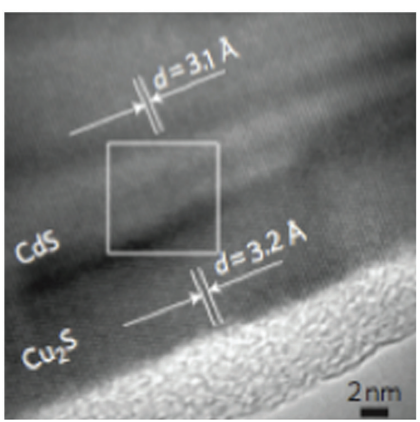

Fig. 4 NW-enabled solar cells. (a) ZnO-dye/sensitized solar cell; (b) large-area Si microwire array for photovoltaic solar cells; (c) aligned Si NW arrays for solar cells; (d) MOVPE-grown large-area InP NW arrays; (e) MBE-grown GaAs NW single solar cells; (f) solution-phase Cu-doped CdS NWs. Adapted with permission from the original versions in refs. [38-45]. Copyright () 2005, 2008, 2009, 2010, 2011, Nature, American Institute of Physics, American Association for the Advancement of Science, American Chemical Society.

et al. have investigated arrays of Si NWs with radial p-n junctions (Fig. 4c) and demonstrated efficiencies of $\sim 0.5 \%$ [42]. Using MOVPE, researchers in Japan have succeeded in synthesizing periodic arrays of doped InP core-shell NWs (Fig. 4d) over $\sim 20 \mathrm{~mm}^{2}$ area and demonstrated an efficiency of $3.4 \%$ [43]. Further demonstrating the diversity of semiconductor NW growth processes, MBE was used to synthesize $\sim 200 \mathrm{~nm}$ GaAs NWs (Fig. 4e) with a radial $p$-i-n geometry and resulting devices showed efficiencies of $\sim 4.5 \%$ [44]. Finally, the Yang group recently demonstrated low-temperature, solution-phase doping of $\mathrm{CdS}$ NWs with $\mathrm{Cu}$ (Fig. 4f) to form active devices with good electrical properties, $V_{\text {oc }}=0.6 \mathrm{~V}$, and efficiencies of $5.4 \%$ [45].

The aforementioned represent a brief survey of several of the research accomplishments in the field of nano-enabled solar cells. They also represent a distinct approach, one based on growth of large-area NW or microwire arrays with subsequent fabrication of device junctions, contacts, and passivation in a top-down [46] manner. In principle, this approach has several benefits including: (1) device fabrication is readily scalable to large-area devices/modules, and (2) vertically oriented wires offer long-optical path lengths and photonic effects can persist in periodic arrays.

\section{Synthesis and assembly}

The Lieber group has pioneered an alternate approach to realizing breakthrough nanoscale functional devices. Often termed the bottom-up approach [47, 48], it simply relies on synthesis of optimized and differentiated nanoscale elements and the subsequent assembly of these into a complex multicomponent device. In fact, Nature has followed such a paradigm to elicit highly complex and functional systems from relatively 
simple building blocks. Amongst the advantages [49] of this approach, several key points are: (1) the ability to differentiate structure and function at the synthesis and assembly stage, (2) the ability to incorporate materials with distinct chemical compositions at the micro-, nano-, and atomic length-scales, (3) the use of in situ processes to achieve high quality and precise control over material properties, and (4) the ability to use new substrates and form factors.

Realization of functional and integrated nanosystems involves three key steps (Fig. 5). First, one must synthesize a comprehensive toolkit of nanoscale building blocks with precisely controlled and tunable chemical compositions, structures, sizes, and morphologies. Second, synthetic differences among these structures must be linked to well-defined electronic/optical properties. As a result, it is critical to study and understand the fundamental properties of these nanoscale elements and explore their limits as functional devices. This link, when properly formed, is the basis of a feedback loop whereby new properties are discovered or evolved, leading to further design of new materials. Third, the development of hierarchical assembly strategies will be key to organization of these building blocks into highly integrated nanosystems with predictable and versatile functions.

\section{Axial and radial nanowire solar cells}

The Lieber group has previously demonstrated the key structural and compositional variations needed to explore the axial and radial PV motifs [50]. Specifically, a critical breakthrough in the development of NW building blocks began with demonstration of controlled growth of axial [22, 51] and radial heterostructures $[25,52]$, where the composition and/or doping was modulated down to the atomic level along or perpendicular to the NW axes, respectively.

For axial $p-i-n$ SiNWs, dopant modulation is achieved by switching dopant precursor gases at appropriate times during NW elongation [53, 54]. Field-emission scanning electron microscopy (SEM) images indicate that the as-grown axial $p$-i-n SiNWs are straight, have smooth surfaces, and a uniform diameter with $<1 \%$ deviation along the typical $25 \mu \mathrm{m}$ length (Fig. 6a, top). Independently calibrated growth rates for each segment of SiNW growth obtained on homogeneous $p$-, $i$-, and $n$-type SiNWs prepared under similar conditions have shown that the lengths of the SiNWs are consistent with overall growth times [55]. In addition, the uniform diameters demonstrate that axial growth is the predominant process under these experimental conditions. In order to verify successful encoding of designed $p$-i-n structures, the SiNWs were selectively etched in potassium hydroxide solution, where the single-crystal SiNW radial etching rate, $\mathrm{R}$, goes as $\mathrm{R}_{i}>\mathrm{R}_{p}>\mathrm{R}_{n}$. SEM images of etched $p-i-n$ SiNW structures (Fig. 6a bottom) show clear delineation of the individual regions of the

a

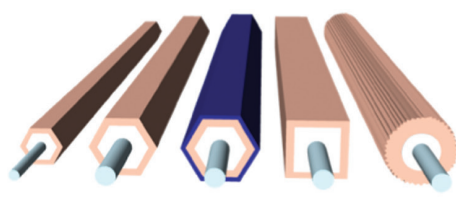

b

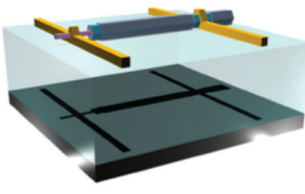

C

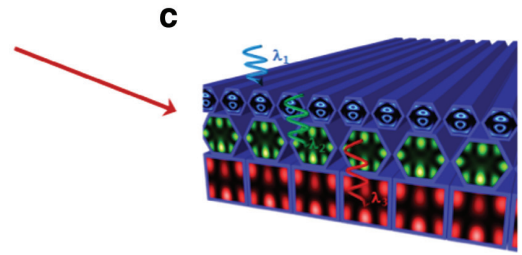

Fig. 5 Bottom-up design of NW-based solar cells. (a) Schematic of NW building blocks (blue: $p$-type core, beige: $n$-type shells); (b) schematic of typical Si core/shell NW device on arbitrary substrate with integrated back reflector; (c) Schematic of a layered solar cell architecture incorporating distinct NWs whose morphologies are optimized to absorb a peak wavelength of light. Reprinted with permission from ref. [62]. Copyright @ 2013, Royal Society of Chemistry. 


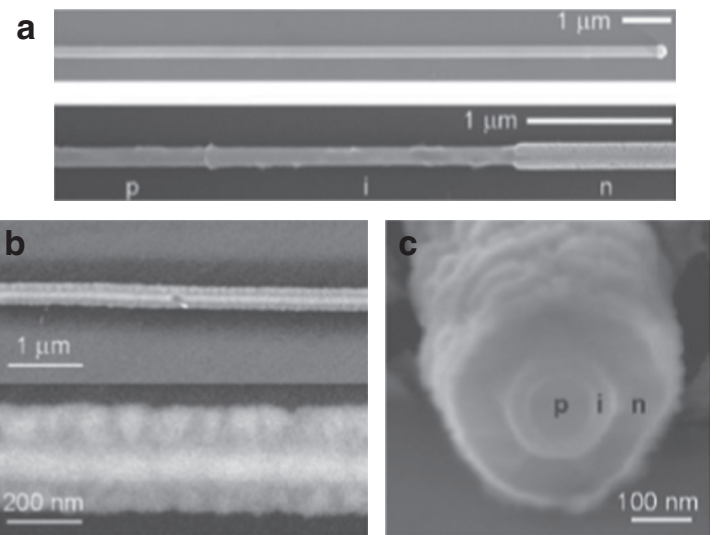

Fig. 6 Synthesis and characterization of NWs for PV. (a) SEM images of $p-i-n$ axial SiNW before (upper panel) and after (lower panel) wet-chemical etching; (b) SEM images of the $p$-i-n radial SiNW at two different magnifications; (c) cross-section SEM image of radial SiNW after wet-chemical etching from a cleaved end. Adapted with permission from the original versions in refs. $[14,55]$. Copyright $\odot$ 2007, 2008, Nature, American Chemical Society.

diode structure, which can be referenced to the Au nanocluster catalyst at the end of the last (n-type) segment [55]. Finally, the SiNW etching profile follows the order in which dopants were introduced during synthesis: first boron for $p$-type, no dopant for $i$-type, and then phosphorous for $n$-type.

For the radial structure, $p$-core growth proceeds as for the axial case and is followed by deposition of $i$ and $n$-shells at higher temperature and lower pressure to inhibit axial elongation of the SiNW core during the radial growth [14]. SEM images of a typical $p-i-n$ coaxial SiNW recorded in the back-scattered electron imaging mode (Fig. 6c) highlight several key features. First, the uniform contrast of the NW core is consistent with a single-crystalline structure expected for SiNWs obtained by the VLS method [17]. Second, contrast variation observed in the shells is indicative of a polycrystalline structure with grain size on the order of 30-80 nm. HRTEM images confirm that the NW shells are indeed polycrystalline. We note here and later comment in greater detail, that this nanocrystalline shell structure may enhance light absorption by the NWs. In analogy with the etching technique used to delineate the axial $p$-i-n diode regions, an SEM image of the cross-section of a radial $p$-i-n SiNW (Fig. 6d) clearly reveals the distinct $p$-core and $i$ - and $n$-shell portions of the structure. Together, these observations show that our designed synthesis can reliably yield axial and radial $p-i-n$ SiNWs with controlled encoding of the length (thickness), dopant profile, and material morphology within different regions. Control of these parameters is, of course, the prerequisite for producing well-defined diode structures necessary for investigating PV devices.

The electrical transport properties of the axial and radial $p-i-n$ SiNW devices have also been characterized by current-voltage $(I-V)$ measurements under dark conditions at room temperature. Dark $I-V$ characteristics recorded from axial $p$-i- $n$ devices with $i$-region lengths of 0 , 2, and $4 \mu \mathrm{m}$ are shown in Fig. 7a [55]. Overall, these data show well-defined current rectification, which is characteristic of the diode structure, and a current onset in forward bias at ca. $0.6 \mathrm{~V}$. The onset value is typical for a $p-n$ Si diode with the built-in potential being established as the difference between the Fermi energies in the $p$ - and $n$-type regions [56]. $I-V$ curves recorded across the $p$ - or $n$-segments alone in $p$-i-n SiNW devices containing multiple contacts (upper inset, Fig. 7a) showed linear behavior (lower inset, Fig. 7a), thus confirming that the contacts are ohmic to the $p$ - and $n$-type segments, and that current rectification is due solely to the built-in electric field across the $p-n$ or $p-i-n$ junction. We also note that the reverse bias leakage current $(V<-1 \mathrm{~V})$ is largest for the $p-n$ SiNW diodes. This leakage can be attributed to larger interfacial recombination in the $p$ - $n$ diodes that is reduced with inclusion of an $i$-region in the $p$-i-n SiNWs.

Dark $I-V$ curves obtained from radial $p-i-n$ devices (Fig. $7 \mathrm{~b}$ ) likewise exhibit characteristics indicative of formation of good diodes [14]. Linear $I-V$ curves from core-core (p1-p2) and shell-shell (n1-n2) configurations demonstrate that ohmic contacts are made to both core and shell portions of the NWs, with the latter 

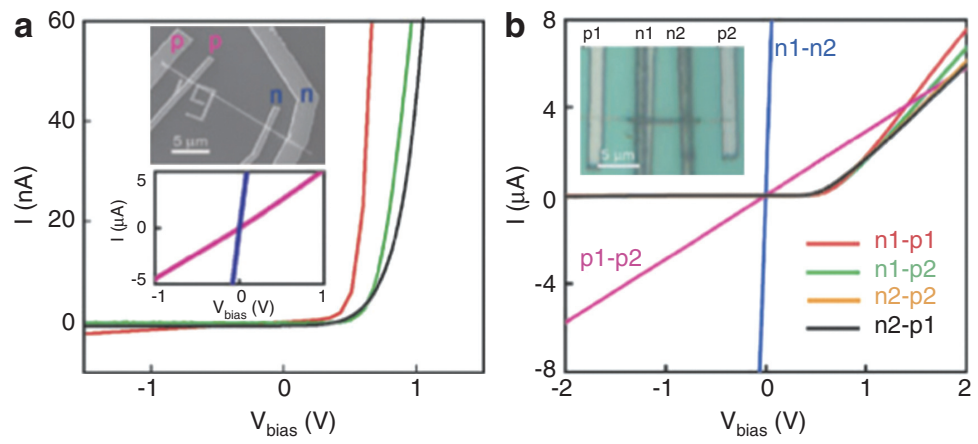

Fig. 7 Dark $I-V$ characteristics of SiNW devices. (a) data recorded from axial $p$-i- $n$ SiNW devices with different $i$-region lengths; red, green, and black curves correspond to $i$-segment lengths of 0,2 , and $4 \mu \mathrm{m}$, respectively. Upper inset: SEM image of $p$-i-n SiNW device with $i$-length of $2 \mu \mathrm{m}, p$ - (pink) and $n$ - (dark blue) contacts marked. Lower inset: $I-V$ curves recorded from two $p$ - (pink) and $n$ - (dark blue) contacts; (b) $I-V$ curves from coaxial $p$ - $i-n$ device. Inset: optical microscope image of the device. Adapted with permission from the original versions in refs. [14, 55]. Copyright $\odot$ 2007, 2008, Nature, American Chemical Society.

showing higher conductance versus the core. Significantly, the highly conductive $n$-shell will reduce or eliminate potential drop along the shell, thereby enabling uniform radial carrier separation and collection when illuminated [14, 39]. $I-V$ curves recorded from different core-shell contact geometries show rectifying behavior, and demonstrate that the $p-i-n$ radial SiNWs behave as well-defined diodes.

The photovoltaic properties of the axial and radial $p-i-n$ SiNW diodes were characterized under standard conditions of 1-sun (100 mW/ $\left.\mathrm{cm}^{2}\right)$ AM1.5G illumination. Representative light $I-V$ data for $p-n, p-i-n i=2 \mu \mathrm{m}$, and $p-i-n i=4 \mu \mathrm{m}$ devices (Fig. 8a) yield open-circuit voltage, $V_{\mathrm{OC}}$, and short-circuit current, $I_{\mathrm{SC}}$, values of 0.12 $\mathrm{V}$ and $3.5 \mathrm{pA}, 0.24 \mathrm{~V}$ and $14.0 \mathrm{pA}$, and $0.29 \mathrm{~V}$ and $31.1 \mathrm{pA}$, respectively. The results reveal a systematic improvement in both $V_{\mathrm{OC}}$ and $I_{\mathrm{SC}}$ with increasing $i$-segment length with the largest increase in $V_{\mathrm{OC}}$ observed in moving from the $p-n$ to $p$-i-n structural motif. Qualitatively, the improvement of $V_{\mathrm{OC}}$ is consistent with the reduction of leakage currents upon inclusion of the $i$-segment. Likewise, the systematic increase in $I_{\mathrm{SC}}$ as a function of increasing $i$-segment length is consistent with previous studies of SiNW avalanche photodiodes [54], which identified the intrinsic region as the most optically sensitive device region. The fill-factor, FF, for the $i=4 \mu \mathrm{m}$ device is $51 \%$ and yields a maximum power output per NW of $4.6 \mathrm{pW}$. This value is approximately 15 times smaller than that achievable at 1-sun illumination using radial $p-i-n$ SiNW devices [14], (see below) but is still $\sim 1000$ times larger than that achieved with single piezoelectric NWs [57].

In addition, the AM1.5G efficiency, $\eta$, based on the projected active area of the $i=4 \mu \mathrm{m}$ device is $0.5 \%$, which is similar to the $0.46 \%$ value reported for single SiNWs with metal/semiconductor junctions [58], but considerably smaller than the $3.4 \%$ achieved in radial SiNW devices [14]. The apparent short-circuit current density $\left(U_{\mathrm{SC}}\right)$ calculated based on the projected active area [14] is $\sim 3.5 \mathrm{~mA} / \mathrm{cm}^{2}$, slightly smaller than that reported for rectifying single metal/SiNW photovoltaic devices $\left(\sim 5.0 \mathrm{~mA} / \mathrm{cm}^{2}\right)$, although the NW diameter in the latter was $4 \times$ larger, $\sim 1 \mu \mathrm{m}$ [58].

The axial $p-i-n$ SiNW photovoltaic devices were further characterized by measurement of temperaturedependent device photovoltaic characteristics. $V_{\mathrm{OC}}$ and $I_{\mathrm{SC}}$ data for a $p-i-n(i=4 \mu \mathrm{m})$ SiNW device are shown in Fig. 8b [55]. $V_{\text {oc }}$ exhibited a well-defined linear dependence on temperature with a slope of $-2.97 \mathrm{mV} / \mathrm{K}$. Extrapolation of $V_{\text {oc }}$ to the $0 \mathrm{~K}$ limit yielded a value of $1.12 \mathrm{~V}$ that is in agreement with the band-gap of singlecrystalline $\mathrm{Si}$ [56]. In addition, $I_{\mathrm{sc}}$ was found to increase weakly with temperature due to a decrease in bandgap with increasing temperature [59-61]. These low-temperature data further confirm that insertion of the $i$-segment is critical to improving the junction quality.

The PV properties of the $p-i-n$ radial SiNW diodes were characterized under identical conditions [14]. $I-V$ data recorded from one of the better devices (Fig. 8c) yielded a $V_{\mathrm{OC}}$ of $0.260 \mathrm{~V}, I_{\mathrm{SC}}$ of $0.503 \mathrm{nA}$, and FF of $55.0 \%$. The maximum power output $\left(P_{\max }\right)$ for the SiNW device at 1-sun is $\sim 72 \mathrm{pW}$, approximately 15 times 

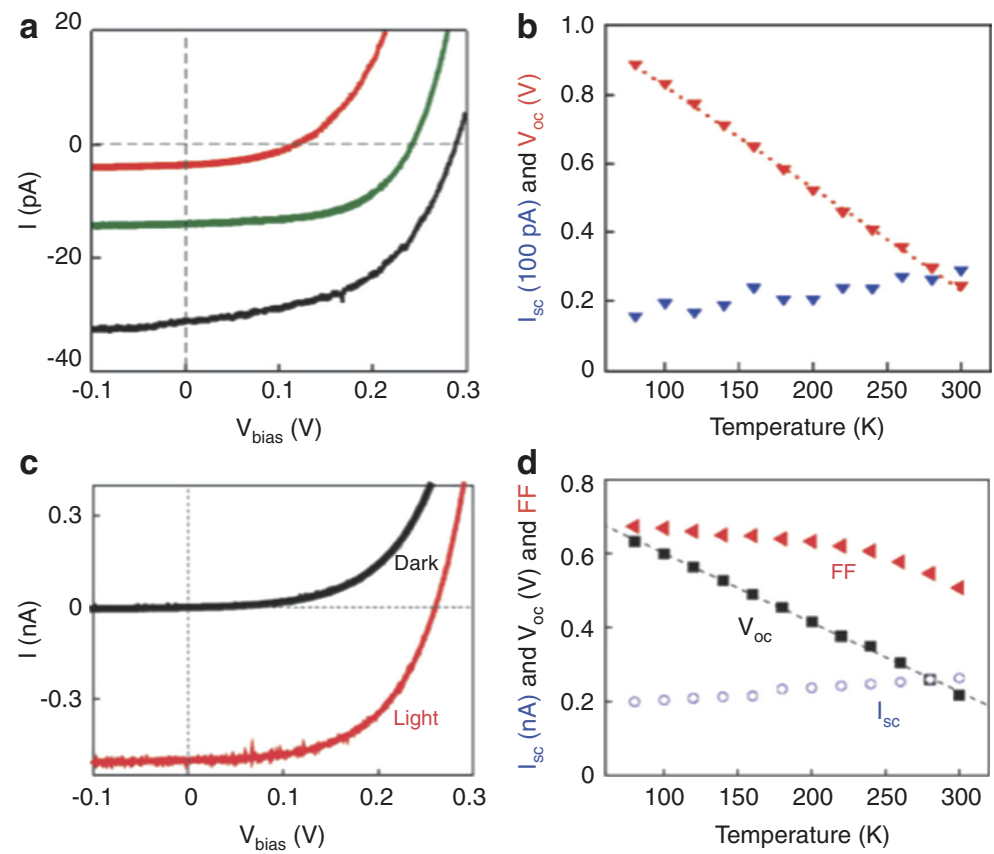

Fig. 8 Photovoltaic and temperature dependent measurement of SiNW devices. (a) Light $I-V$ curves recorded from axial $p$-i-n SiNW, $i$-length $=0$ (red), 2 (green), and 4 (black) $\mu \mathrm{m}$ devics with illumination intensity of $100 \mathrm{~mW} / \mathrm{cm}^{2}$, AM1.5G; (b) plots of $V_{\text {oc }}$ (red) and $I_{\mathrm{sc}}$ (blue) vs. temperature for a $p-i-n(i=4 \mu \mathrm{m})$ device; (c) dark and light $I-V$ curves of coaxial SiNW device;

(d) temperature-dependent data from a coaxial device, where the red triangle, black square, and blue circle correspond to $\mathrm{FF}$, $V_{\text {oc }}$, and $I_{\text {sc }}$, respectively. Adapted with permission from the original versions in refs. [14, 55]. Copyright @ 2007, 2008, Nature, Nature Publishing Group, ACS Publications.

larger than for the axial $p$-i-n structure [55]. The $J_{\mathrm{SC}}$ calculated using the projected area of the radial NW structure is $23.9 \pm 1.2 \mathrm{~mA} / \mathrm{cm}^{2}$ [14]. The large $J_{\mathrm{SC}}$ in the radial NW device implies substantial absorption across the solar spectrum and efficient carrier collection. Such absorption is consistent with the nanocrystalline shell structure of the NWs and previous studies of polycrystalline thin films [61], although the detailed nature of absorption will require further investigation. Finally, the apparent PV efficiency of this device was $3.4 \pm 0.2 \%$.

In addition, the temperature dependence of $I_{\mathrm{SC}}, V_{\mathrm{OC}}$, and $\mathrm{FF}$ were characterized to understand better the behavior of the radial SiNW PV devices (Fig. 8d) [14]. Consistent with the observations in the axial structure, $I_{\mathrm{SC}}$ increases slightly with increasing temperature, as expected for increased light absorption due to decreasing band-gap with elevated temperature [59]. Second, $V_{\text {oC }}$ exhibits a substantial linear increase with decreasing temperature, where the slope $\left(d V_{\text {oc }} / d T\right)$ of $-1.9 \mathrm{mV} / \mathrm{K}$ is close to the value $(-1.7 \mathrm{mV} / \mathrm{K})$ calculated in single-crystalline $\mathrm{Si}$ solar cells [60]. Interestingly, $V_{\text {oC }}$ does not extrapolate to the single-crystal $\mathrm{Si}$ band-gap as expected for a structure consisting of single-crystal core and polycrystalline shells.

\section{Multishell nanowires as tunable elements for next-generation pv}

SiNWs could enable low-cost and efficient photovoltaics, though their performance has been limited by poor electrical characteristics, as seen above, and an inability to tune absorption properties. As discussed above, single axial and radial NW devices posed two major challenges: (1) SiNW solar cells exhibited very poor electrical performance, and (2) SiNW solar cells exhibited weak or poorly tailorable absorption. The first problem relates to the low $V_{\text {oC }}(\sim 0.2 \mathrm{~V}$ ) and high leakage current ( $>2000 \mathrm{fA}$ ) observed for radial devices. While tolerable if such a device were configured as standalone nanoscale power source, such poor electrical characteristics would preclude the attainment of higher efficiencies and the integration of such elements 
into large-area arrays for multicomponent solar cells. The second problem relates less to the low $J_{\mathrm{sc}}$ attained for the axial device and more to the fact that the radial device, despite high $J_{\mathrm{sC}}$, exhibits a bulk-like absorption external quantum efficiency (EQE) spectrum. Far from a fine point, this fact would preclude a radial device from ever yielding anything better than bulk absorption and thus would be unable to surpass the limit of current state-of-the-art crystalline Si solar cells. This limitation runs counter to one of the stated goals of impact for nano-enabled next-generation solar cells, namely, that they attempt to achieve power conversion efficiencies $>20 \%$.

\section{Enhanced and tunable absorption in radial NWs}

We recently synthesized high-quality core/multishell NW devices exhibiting $\sim 0.5 \mathrm{~V} V_{\mathrm{OC}} \mathrm{s}$ and $J_{\mathrm{SC}}$ values of 8-10 $\mathrm{mA} / \mathrm{cm}^{2}$ [13]. The latter values are substantially larger than expected for an equivalent thickness of bulk $\mathrm{Si}$ [63]. Data obtained on NW devices with lengths spanning over 1 order of magnitude exhibit linear scaling of $I_{\mathrm{SC}}$ and nearly constant $J_{\mathrm{SC}}$, suggesting that the high $J_{\mathrm{SC}}$ values are intrinsic to these nanoscale structures and are not due to extrinsic factors such as scattering by contact electrodes [14]. To understand the origin of these large $J_{\text {sc }}$ values, we carried out wavelength-dependent photocurrent measurements and finite-difference time-domain (FDTD) simulations on individually characterized core/multishell NW devices. A representative curve of the absolute EQE vs. wavelength acquired for a p/in NW device (Fig. 9a, black curve) and the corresponding simulated EQE spectrum (Fig. 9a, red dashed curve) demonstrate several important points. First, the experimental and simulated spectra are highly structured, with very good agreement in the peak

a

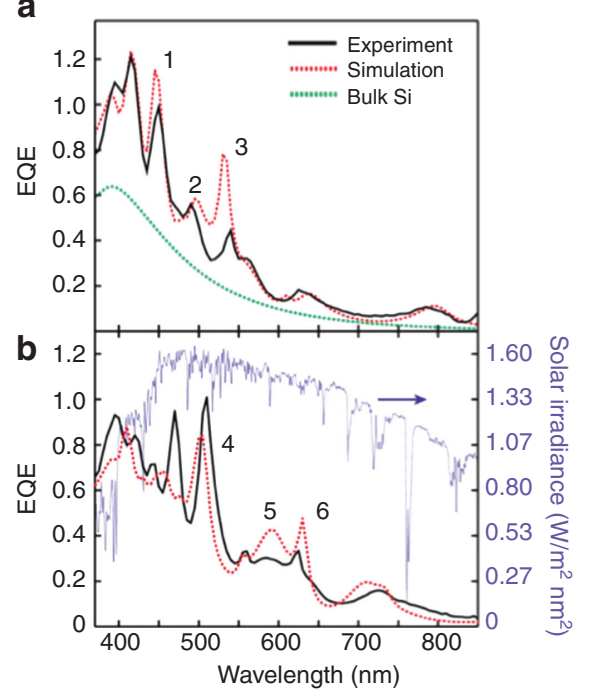

C

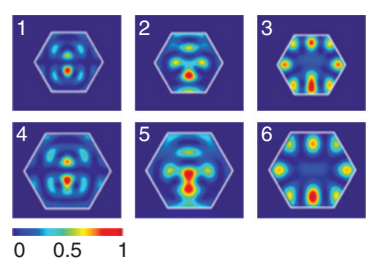

d

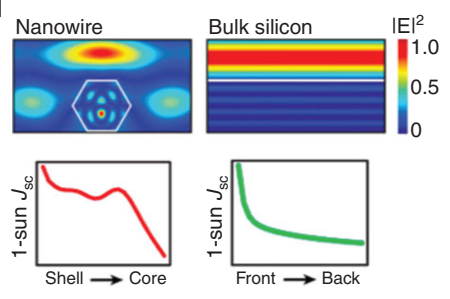

Fig. 9 Enhanced and tunable absorption in NWs. (a) EQE as a function of wavelength for a p/in NW (black curve) and simulated EQE spectrum (dashed red curve) produced with no adjustable parameter other than the size of the NW (height of $240 \mathrm{~nm}$ ). Dashed green curve shows the simulated spectrum for top $240 \mathrm{~nm}$ of planar bulk Si; (b) EQE spectrum (black curve) of a p/pin NW compared to the irradiance of the AM1.5G solar spectrum (dashed blue curve). Dashed red curve corresponds to simulated EQE calculated with no other adjustable parameter besides the NW size (height of $305 \mathrm{~nm}$ ); (c) FDTD simulations of resonant mode spatial profiles for $p /$ in (profiles 1-3) and p/pin (profiles 4-6) structures. Labeled profiles correspond to the EQE peaks labeled identically within Fig. 9a,b and profiles in each column correspond to the same mode excited within both structures. All profiles are for TM polarizations and use a linear color scale representing absorption (not electric field intensity) within the mesoscopic structures. Resonant modes labeled 3 and 6 correspond to whispering-gallery type modes while all others correspond to Fabry-Perot resonances; (d) plot of electric field intensity for plane wave $(\lambda=445 \mathrm{~nm}$ ) interacting with a NW (left) and bulk $\mathrm{Si}$ (right). White line defines outline of NW and top surface of bulk Si. Beneath are plots of total $J_{\mathrm{sc}}$ as a function of position inside the NW or bulk Si. Reprinted with permission from ref. [13]. Copyright @ 2012, National Academy of Sciences, USA. 
positions. Significantly, from analysis of the simulations and polarization-dependent experiments we can assign peaks in EQE to wavelength-dependent Fabry-Perot (Fig. 9 labels 1, 2, 4, and 5) and whispering-gallery (Fig. 9 labels 3 and 6) resonant absorption modes within the hexagonal NW structure.

Second, there is excellent correspondence in absolute EQE amplitudes between experiment and simulation, where the full-field FDTD simulations are executed without adjustable parameters and assume that internal quantum efficiency (IQE) has value of unity. This agreement in amplitudes further supports the use of NW projected area in calculation of current densities, and moreover, indicates that charge recombination is minimized in our new $\mathrm{p} /$ in and $\mathrm{p} / \mathrm{pin}$ core/multishell structures. Third, the EQE spectrum approaches and even exceeds unity for wavelengths in the range of $400-500 \mathrm{~nm}$. EQE values greater than unity are possible because of an optical antenna effect $[64,65]$, in which the absorption cross-section of the NW exceeds its physical cross-section for some photon energies. This phenomenon is a purely classical effect caused by the sub-wavelength diameter of the NW and is quantitatively reproduced by the FDTD simulations.

To illustrate how rational synthetic design can be used to tune absorption within our NW photovoltaic elements, we examined a larger diameter (305 vs. $240 \mathrm{~nm}$ ) p/pin NW device. The measured and simulated absolute EQE spectra for the p/pin device (Fig. 9b) exhibit several features differentiating them from the corresponding spectra discussed above for the p/in NW device. First, peaks 4-6 in the p/pin spectrum (Fig. 9b), which correspond to the same modes (Fig. 9c) represented by peaks 1-3 in the p/in spectrum (Fig. 9a), are substantially red-shifted. Second, the $\mathrm{p} /$ pin device exhibits a larger number of peaks in the experimental and simulated EQE spectra. Third, the p/pin experimental spectrum (Fig. 9b) exhibits two peaks between 470 and $520 \mathrm{~nm}$ with near-unity amplitudes. These two peaks correspond to resonant modes localized near the core-shell interface (Fig. 9c: label 4). We suggest that the higher than predicted EQE values may result from enhanced absorption within the first $\sim 20 \mathrm{~nm}$ of the polymorphic $\mathrm{p}$-shell surrounding the p-core, although future studies will be needed to confirm and potentially exploit these differences in detail. The observed redshifted modes with enhanced absorption are also better matched in frequency to the maximum irradiance of the solar spectrum (Fig. 9b, blue dashed curve). This observation explains the $\sim 40 \%$ larger $J_{\mathrm{sC}}$ of $\mathrm{p} / \mathrm{pin}$ NWs compared to $\mathrm{p} /$ in NWs and underscores our ability to optimize device performance through synthetic tuning of nanostructures.

We also compared the experimental and simulated optical properties of a p/in core/multishell NW (Fig. 9a) to an equivalent thickness of bulk Si. The simulated EQE spectrum for $240 \mathrm{~nm}$ of bulk Si (Fig. 9a, dashed green line) is featureless and shows substantially lower amplitude than the p/in NW. The origin of the differences in EQE spectra can be understood by comparing the interaction of light with both structures. For example, a plot of the electrical field intensity for an incident plane wave with a wavelength of $445 \mathrm{~nm}$ (Fig. 9d) shows a strong resonant mode excited within the NW, but the profile of electric field within an equivalent thickness of bulk Si is featureless. These modes in the NWs lead to substantial and structured $J_{\mathrm{SC}}$ contribution from the shell through much of the core, while bulk Si exhibits the standard exponential decay as function of thickness (Fig. 9d). Significantly, the spatial localization and intensity of resonant modes could be adjusted by tuning the morphology of the NW cavity.

\section{Scaling properties and progress towards assembly}

The studies discussed above demonstrate substantial improvement in the photovoltaic properties of SiNWs at the single NW level. To determine whether $J_{\mathrm{SC}}$ could be improved further, we fabricated NW devices on transparent quartz substrates and measured $J-V$ characteristics with and without a silver metal back-side reflector (BSR), as shown in Fig. 10a. Without the BSR, a representative single p/pin device (red line) yields $J_{\mathrm{SC}}=9.8$ $\mathrm{mA} / \mathrm{cm}^{2}$ and an overall conversion efficiency, $\eta$, of $3.2 \%$. Significantly, the same device with a BSR yields $J_{\mathrm{SC}}=17.0 \mathrm{~mA} / \mathrm{cm}^{2}$ and $\eta=5.9 \%$. The substantial increase in efficiency achieved with the BSR thus suggests one straightforward route to mitigate incomplete absorption and thereby enables efficient NW-based solar cells.

In addition, the ability to maintain high-quality devices from very long $(\sim 50 \mu \mathrm{m})$ NWs bodes well for scaling to larger-area solar cells from assembled arrays of NWs. With a longer exposed shell, a larger surface 

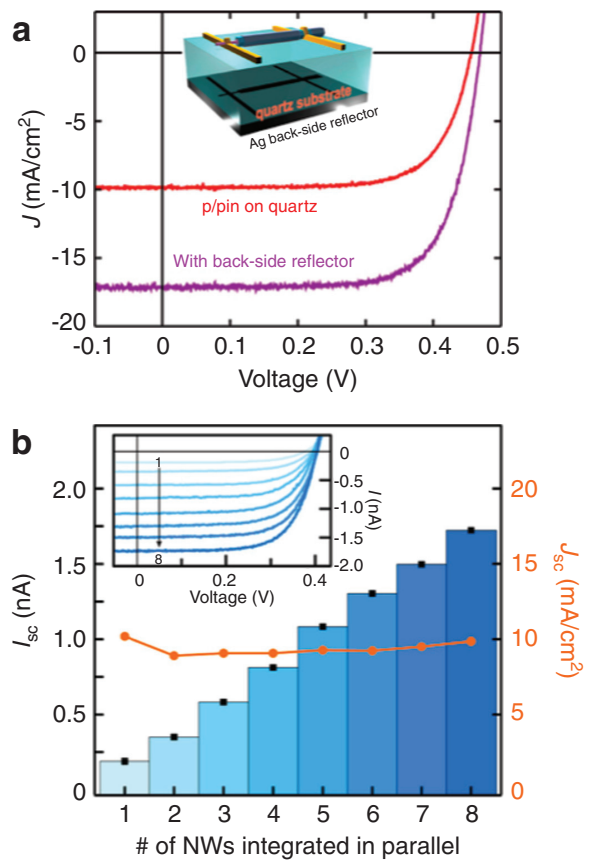

Fig. 10 Integration of NW components for efficient solar cells. (a) Current density-voltage ( $-V$ ) characteristics for a p/pin device without (red curve) and with a Ag back-side reflector (purple curve) under 1-sun illumination. Inset: schematic of the device layout on quartz; (b) linear scaling of $I_{\mathrm{sc}}$ (black squares and shaded blue bars) and preservation of $J_{\mathrm{sc}}$ (orange circles and line) as multiple $\mathrm{p} / \mathrm{pin}$ NWs are integrated in parallel. Inset: light $I-V$ curves for devices consisting of 1-8 NWs connected in parallel. Reprinted with permission from ref. [13]. Copyright @ 2012, National Academy of Sciences, USA.

is made available for light absorption, and a larger $p$-core to $n$-shell contact spacing also means that photolithography will be sufficient to define etch regions and electrical contacts.

While individual NW photovoltaics represent the ideal platform for elucidating fundamental factors affecting device efficiency and can be used to power nanoelectronic elements [14], it is also important to consider the prospects for scaling these to arrays that will be required for solar cells. As an initial step in this direction we interconnected in parallel from 1 to $8 \mathrm{p} /$ pin core/multishell NW elements. Measurements of light $I-V$ data from these small multi-NW devices (inset, Fig. 10b) show good "cell" characteristics are maintained (e.g., $V_{\text {oc }}$ and FF are nearly constant) with increasing number of NW elements. Indeed, the FF for the $8 \mathrm{NW}$ device, $69.3 \%$, is similar to the best value we achieved on single NW elements, $68.0 \%$. Moreover, and central to the potential for scaling, we observe a step-wise increase in $I_{\mathrm{SC}}$ from 191 to $1723 \mathrm{pA}$ as the number of NWs in a device is increased from 1 to 8, respectively (Fig. 10b). Importantly, this increase in absolute $I_{\mathrm{SC}}$ is also accompanied by preservation of $J_{\mathrm{SC}}$ to within $3 \%$ of that for the best single NW device. We believe these results show that assembly and large-scale integration could be a viable strategy for using our core/ multishell NW building blocks, although future studies will be required to expand to larger arrays. Such work should be able to exploit reported advances in assembly of dense parallel NWs on the micrometer to several inch scale [66], where the NWs have also exhibited good electronic properties [66, 67].

In line with the paradigm we introduced in Fig. 5, we demonstrate how layering and interconnection of designed coaxial core/multishell NW components can significantly enhance current density and thereby efficiency. To advance this new concept, we assembled vertical stacks of horizontally oriented NW devices. A representative SEM image of the assembled NWs (inset, Fig. 11a) shows the well-aligned vertical stack of two $\mathrm{p} /$ pin NWs on a quartz substrate. Transport measurements for the vertical stack of two parallel-connected $\mathrm{p} /$ pin NWs yield a $J_{\mathrm{SC}}$ of $14.0 \mathrm{~mA} / \mathrm{cm}^{2}$ (Fig. 11b), which represents a $1.4 \times$ increase compared to the best single p/pin NW device. Significantly, the experimental EQE spectrum for the stacked double NW device (Fig. 11a) shows that nearly all peaks from 380 to $700 \mathrm{~nm}$ coincide in frequency with those for a single NW device, and that the EQE amplitudes are increased by a factor of 1.0-2.0 across this wavelength range. These increased 

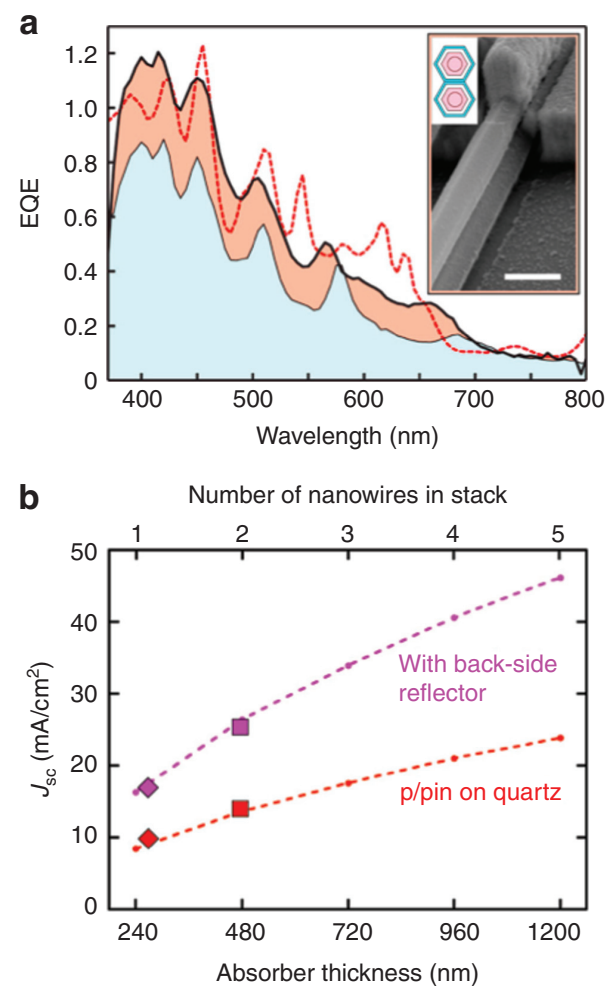

Fig. 11 Vertical assembly of layered NWs for efficient solar cells. (a) Experimental EQE spectrum for double-NW vertical stack (thick black line) and for single NW (thin black line) device. Both devices are on quartz without back-reflectors. Simulated EQE spectrum for the double-NW stack (dashed red line) using NWs with a Si height of $250 \mathrm{~nm}$. Inset: SEM of a device consisting of two NWs stacked on quartz substrate and electrically connected in parallel; scale bar, $500 \mathrm{~nm}$; (b) experimental current densities for a single NW device (diamonds) and stacked double NW device (squares) under c. Red markers show data without a Agmetal BSR and purple markers data with the Ag-metal BSR. Dashed red and purple curves denote simulated current densities for stacked NWs with increasing number of NWs in the $\operatorname{stack}(N=1,2,3,4,5)$ without and with a BSR, respectively. Reprinted with permission from ref. [13]. Copyright () 2012, National Academy of Sciences, USA.

amplitudes account for the observed $40 \% J_{\mathrm{SC}}$ enhancement, and notably, FDTD simulations reproduce the EQE enhancement for the double stack configuration and predict a comparable $41 \%$ increase in $J_{\text {SC }}$.

Simulations also show that the resonant modes present in a single or, in this case, the top NW are preserved in the bottom NW, and this effect permits the broadband enhancement observed in the experimental and simulated EQE spectra for the double-NW stack. In addition, we explored the scaling of our new concept by simulating NW stack structures with up to five equal-diameter NWs and a total thickness of $1.2 \mu \mathrm{m}$ (Fig. 11b). Comparison of the $J_{\mathrm{SC}}$ and $\mathrm{EQE}$ for the 5-NW stack and a single microwire with a diameter of the same height shows that the 5-NW stack is predicted to have $26 \%$ higher $J_{\mathrm{SC}}$ and 2-fold higher EQE at $620 \mathrm{~nm}$, with the EQE of the stack exceeding that of the microwire for most wavelengths. Notably, simulations show that a stack of five NWs could yield $J_{\mathrm{SC}}$ values of 24 and $>40 \mathrm{~mA} / \mathrm{cm}^{2}$ without and with a BSR, respectively, values that could yield power-conversion efficiencies $>15 \%$.

\section{Conclusions and outlook}

In the future, studies should continue to focus on the synthesis of new nanoscale materials $[13,68,69]$ and also explore innovative methods for the assembly of large-area nanostructure arrays for photovoltaic applications. Significant challenges and opportunities exist for the development of assembled NW photovoltaic arrays. The ability to pattern arrays of NWs over large areas, at high density, and with robust control of their 
periodicity would constitute a breakthrough. Furthermore, NW design [70] and synthesis efforts should minimize the size and doping heterogeneity of NWs, because variations in such parameters limit the overall power conversion efficiency of the system [71]. Finally, though the systems cost of an assembled NW photovoltaic cell is difficult to quantify at present, synthetic advances, use of cheaper catalysts, and adoption of lower-temperature processes will drive cost reductions. Ultimately, the assembly and large-scale integration of diverse NW building blocks could become a viable strategy for development of efficient and cost-effective next-generation solar cells.

\section{References}

[1] Y. Cui, Q. Q. Wei, H. K. Park, C. M. Lieber. Science 293, 1289 (2001).

[2] Y. Cui, C. M. Lieber. Science 291, 851 (2001).

[3] Y. Cui, Z. H. Zhong, D. L. Wang, W. U. Wang, C. M. Lieber. Nano Lett. 3, 149 (2003).

[4] C. M. Lieber. Nano Lett. 2, 81 (2002).

[5] D. D. D. Ma, C. S. Lee, F. C. K. Au, S. Y. Tong, S. T. Lee. Science 299, 1874 (2003).

[6] F. Patolsky, G. F. Zheng, O. Hayden, M. Lakadamyali, X. W. Zhuang, C. M. Lieber. Proc. Natl. Acad. Sci. USA 101, 14017 (2004).

[7] F. Patolsky, G. F. Zheng, C. M. Lieber. Nanomedicine 1, 51 (2006).

[8] G. F. Zheng, W. Lu, S. Jin, C. M. Lieber. Adv. Mater. 16, 1890 (2004).

[9] Z. H. Zhong, Y. Fang, W. Lu, C. M. Lieber. Nano Lett. 5, 1143 (2005).

[10] T. Cohen-Karni, B. P. Timko, L. E. Weiss, C. M. Lieber. Proc. Natl. Acad. Sci. USA 106, 7309 (2009).

[11] B. Tian, T. Cohen-Karni, Q. Qing, X. Duan, P. Xie, C. M. Lieber. Science 329, 831 (2010).

[12] F. Qian, S. Gradecak, Y. Li, C. Y. Wen, C. M. Lieber. Nano Lett. 5, 2287 (2005).

[13] T. J. Kempa, J. F. Cahoon, S.-K. Kim, R.W. Day, D.C. Bell, H.-G. Park, C. M. Lieber. Proc. Natl. Acad. Sci. USA 109, 1407 (2012).

[14] B. Tian, X. Zheng, T. J. Kempa, Y. Fang, N. Yu, G. Yu, J. Huang, C. M. Lieber. Nature 449, 885 (2007).

[15] R. S. Wagner. Whisker Technology, John Wiley, New York (1970).

[16] B. P. Timko. Ph.D. Dissertation. February, 2009.

[17] A. M. Morales, C. M. Lieber. Science 279, 208 (1998).

[18] J. L. Liu, S. J. Cai, G. L. Jin, S. G. Thomas, K. L. Wang. J. Cryst. Growth 200, 106 (1999).

[19] L. J. Lauhon, M. S. Gudiksen, C. M. Lieber. Philos. Trans. R. Soc. London, Ser. A 362, 1247 (2004).

[20] K. Haraguchi, T. Katsuyama, K. Hiruma, K. Ogawa. Appl. Phys. Lett. 60, 745 (1992).

[21] X. Duan, C. M. Lieber. Adv. Mater. 12, 298 (2000).

[22] M. S. Gudiksen, L. J. Lauhon, J. Wang, D. C. Smith, C. M. Lieber. Nature 415, 617 (2002).

[23] Y. Wu, R. Fan, P. Yang. Nano Lett. 2, 83 (2002).

[24] B. Tian, P. Xie, T. J. Kempa, D. C. Bell, C. M. Lieber. Nat. Nanotechnol. 4, 824 (2009).

[25] L. J. Lauhon, M. S. Gudiksen, C. L. Wang, C. M. Lieber. Nature 420, 57 (2002).

[26] W. Lu, J. Xiang, B. P. Timko, Y. Wu, C. M. Lieber. Proc. Natl. Acad. Sci. USA 102, 10046 (2005).

[27] M. A. Green. Prog. Photovolt.: Res. Appl. 9, 123 (2001).

[28] C. Brabec, MRS Bull. 30, 50 (2005).

[29] G. Conibeer. Mater. Today 10, 42 (2007).

[30] A. Polman, H. A. Atwater. Nat. Mater. 11, 174 (2012).

[31] Y. Li, F. Qian, J. Xiang, C. M. Lieber. Mater. Today 9, 18 (2006).

[32] L. Lauhon, M. Gudiksen, D. Wang, C. M. Lieber. Nature 420, 57 (2002).

[33] M. A. Green. Third Generation Photovoltaics: Advanced Solar Energy Conversion, Springer Science, Boston (2003).

[34] A. Marti, A. Luque (Eds.). Next Generation Photovoltaics: High Efficiency Through Full Spectrum Utilization, Series on Optics and Optoelectronics, Institute of Physics, Bristol (2003).

[35] N.-P. Harder, P. Wurfel. Semi. Sci. Tech. 18, S151 (2003).

[36] A. Luque, A. Marti, A. J. Nozik. MRS Bull. 32, 236 (2007).

[37] W. Shockley, H. J. Queisser. J. Appl. Phys. 32, 510 (1961).

[38] M. Law, L. E. Greene, J. C. Johnson, R. Saykally, P. Yang. Nat. Mater. 4, 455 (2005).

[39] B. M. Kayes, H. A. Atwater, N. S. Lewis. J. Appl. Phys. 97, 114302 (2005).

[40] S. W. Boettcher, J. M. Spurgeon, M. C. Putnam, E. L. Warren, D. B. Turner-Evans, M. D. Kelzenberg, J. R. Maiolo, H. A. Atwater, N. S. Lewis. Science 327, 185 (2010).

[41] M. D. Kelzenberg, S. W. Boettcher, J. A. Petykiewicz, D. B. Turner-Evans, M. C. Putnam, E. L. Warren, J. M. Spurgeon, R. M. Briggs, N. S. Lewis, H. A. Atwater. Nat. Mater. 9, 239 (2010).

[42] E. C. Garnett, P. Yang. J. Am. Chem. Soc. 130, 9224 (2008). 
[43] H. Goto, K. Nosaki, K. Tomioka, S. Hara, K. Hiruma, J. Motohisa, T. Fukui. Appl. Phys. Express 2, 035004 (2009).

[44] C. Colombo, M. Heiss, M. Gratzel, A. Fontcuberta i Morral. Appl. Phys. Lett. 94, 173108 (2009).

[45] J. Tang, Z. Huo, S. Brittman, H. Gao, P. Yang. Nat. Nanotechnol. 6, 568 (2011).

[46] G. M. Whitesides. Small 1, 172 (2005).

[47] C. M. Lieber. MRS Bull. 28, 486 (2003).

[48] J. D. Meindl, Q. Chen, J. A. Davis. Science 293, 2044 (2001).

[49] J. R. Heath, P. J. Kuekes, G. S. Snider, R. S. Williams. Science 280, 1716 (1998).

[50] Y. Li, F. Qian, J. Xiang, C. M. Lieber. Mater. Today 9, 18 (2006).

[51] Y. Wu, J. Xiang, C. Yang, W. Lu, C. M. Lieber. Nature 430, 61 (2004).

[52] F. Qian, Y. Li, S. Gradecak, D. Wang, C. J. Barrelet, C. M. Lieber. Nano Lett. 4, 1975 (2004).

[53] C. Yang, Z. Zhong, C. M. Lieber. Science 310, 1304 (2005).

[54] C. Yang, C. J. Barrelet, F. Capasso, C. M. Lieber. Nano Lett. 6, 2929 (2006).

[55] T. J. Kempa, B. Tian, D. Kim, J. Hu, X. Zheng, C. M. Lieber. Nano Lett. 8, 3456 (2008).

[56] S. M. Sze, K. K. Ng. Physics of Semiconductor Devices, John Wiley, Hoboken (2007).

[57] Z. L. Wang, J. Song. Science 312, 242 (2006).

[58] M. D. Kelzenberg, D. B. Turner-Evans, B. M. Kayes, M. A. Filler, M. C. Putnam, N. S. Lewis, H. A. Atwater. Nano Lett. 8, 710 (2008).

[59] M. A. Green. Prog. Photovolt.: Res. Appl. 11, 333 (2003).

[60] P. Würfel. Physics of Solar Cells, From Principles to New Concepts, Wiley-VCH, Weinheim (2005).

[61] A. Luque S. Hegedus. Handbook of Photovoltaic Science and Engineering, John Wiley, Chichester (2003).

[62] T. J. Kempa, R. W. Day, S.-K. Kim, H. G. Park, C. M. Lieber. Energy Environ. Sci. 6, 719 (2013).

[63] L. G. Jeffery. In Handbook of Photovoltaic Science and Engineering, S. Hegedus, A. Luque (Eds.), pp. 61-112, John Wiley, New York (2004).

[64] L. Cao, J. S. White, J.-S. Park, J. A. Schuller, B. M. Clemens, M. L. Brongersma. Nat. Mater. 8, 643 (2009).

[65] L. Cao, P. Fan, A. P. Vasudev, J. S. White, Z. Yu, W. Cai, J. A. Schuller, S. Fan, M. L. Brongersma. Nano Lett. 10, 439 (2010).

[66] Z. Fan, J. C. Ho, Z. A. Jacobson, R. Yerushalmi, R. L. Alley, H. Razavi, A. Javey. Nano Lett. 8, 20 (2008).

[67] A. Javey, S. W. Nam, R. S. Friedman, H. Yan, C. M. Lieber. Nano Lett. 7, 773 (2007).

[68] S. K. Kim, R. W. Day, J. F. Cahoon, T. J. Kempa, K. Deok, H.-G. Park, C. M. Lieber. Nano Lett. 12, 4971 (2012).

[69] M. J. Bierman, S. Jin. Energy Environ. Sci. 2, 1050 (2009).

[70] J. D. Christesen, X. Zhang, C. W. Pinion, T. A. Celano, C. J. Flynn, J. F. Cahoon. Nano Lett. 12, 6024 (2012).

[71] J. M. Foley, M. J. Price, J. I Feldblyum, S. Maldonado. Energy Environ. Sci. 5, 5203 (2012). 